\title{
Intravesical Migration of Hem-o-Lok Clips Presenting as Vesical Calculus After Robot-Assisted Laparoscopic Radical Prostatectomy
}

\author{
Laxman Swaroop, MS, MCH, Brusabhanu Nayak, MS, MCH, Prem Nath Dogra, MS, MCA, \\ Prabhjot Singh, MS, MCA
}

Department of Urology, Room no-331, Maszid Moth Hostel, Ansari Nagar, All India Institute of Medical Sciences, New Delhi, India (all authors).

\begin{abstract}
Vesical stone formation over migrated Hem-o-lok clips (Weck Surgical Instruments [Teleflex Medical], Durham, North Carolina) after robot-assisted laparoscopic radical prostatectomy is rare. We report our experience in 2 patients who presented with lower urinary tract symptoms after robot-assisted laparoscopic radical prostatectomy and were found to have vesical stones formed over migrated Hem-o-lok clips.
\end{abstract}

Key Words: RALRP, Intravesical clip, Hem-o-lok clip.

Citation Swaroop L, Nayak B, Dogra PN, Singh P. Intravesical migration of Hem-o-lok clips presenting as vesical calculus after robot-assisted laparoscopic radical prostatectomy. CRSLS e2014.00233. DOI: 10.4293/CRSLS.2014.00233.

Copyright (C) 2014 SLS This is an open-access article distributed under the terms of the Creative Commons Attribution-Noncommercial-ShareAlike 3.0 Unported license, which permits unrestricted noncommercial use, distribution, and reproduction in any medium, provided the original author and source are credited.

Address correspondence to: Brusabhanu Nayak, Department of Urology, All India Institute of Medical Sciences, Room No. 331, Maszid Moth Hostel, New Delhi, India Phone: 919814465239, E-mail: brusabhanu@gmail.com

\section{INTRODUCTION}

Robot-assisted laparoscopic radical prostatectomy (RALRP) is a well-established surgical treatment for prostate cancer. ${ }^{1}$ During surgery, various modalities such as suture ligation, bipolar cautery, the Harmonic scalpel (Ethicon Endo-Surgery, Cincinnati, Ohio), and Hem-o-lok clips (Weck Surgical Instruments [Teleflex Medical], Durham, North Carolina) are used at various stages of the procedure to help achieve hemostasis and for ligation of the vas deferens. The use of Hem-o-lok clips is well documented for the lateral pedicles of the prostate during RALRP. At our institute, 300 RALRPs have been performed. We report on 2 cases of intravesical migration of Hem-o-lok clips and their consequences.

\section{CASE 1}

The first patient was a 71-year-old man who was diagnosed with prostate carcinoma (stage T1c) by increased serum prostate-specific antigen level during a routine health checkup. Transrectal ultrasonography-guided 12core biopsy showed adenocarcinoma prostate with a Gleason score of $4+3$ and foci of perineural invasion.
The patient underwent further evaluation with magnetic resonance imaging of the abdomen and pelvis and a bone scan to rule out any metastatic disease. After proper evaluation, he underwent RALRP. Surgery was uneventful. Various modalities including electrocautery, Ligaclip (Ethicon, Somerville, New Jersey), and Hem-o-lok clips were used at various steps for hemostasis. Vesicourethral anastomosis was performed with continuous Vicryl suture (Ethicon). The final histopathology report showed adenocarcinoma with a Gleason score of $4+3$; all resected margins, the bilateral seminal vesicles, and the vas deferens were free of tumor. The urethral catheter was removed 15 days after surgery. After 1 year, the patient developed dysuria with urinary frequency and urgency. He was then evaluated and found to have vesical calculus on ultrasonography of the abdomen and plain radiography of the kidney, ureter, and bladder. He underwent urethrocystoscopy and cystolithotripsy. On urethrocystoscopy, 2 encrusted Hem-o-lok clips were shown to be present inside the urinary bladder. The encrustations were fragmented with a Holmium Laser:YAG laser. After removal of the encrustations, the Hem-o-lok clips were seen. The clips were removed through the urethra (Figs. 1-4). The urethral 
Intravesical migration of Hem-o-lok clips after robot-assisted laparoscopic radical prostatectomy, Laxman S et al.

catheter was removed after 24 hours. The patient was asymptomatic at the most recent follow-up visit.

\section{CASE 2}

The second patient was a 62-year-old man who presented with lower urinary tract symptoms. He was evaluated and diagnosed with organ-confined prostate carcinoma. The serum prostate-specific antigen level was $6.25 \mathrm{ng} / \mathrm{mL}$,

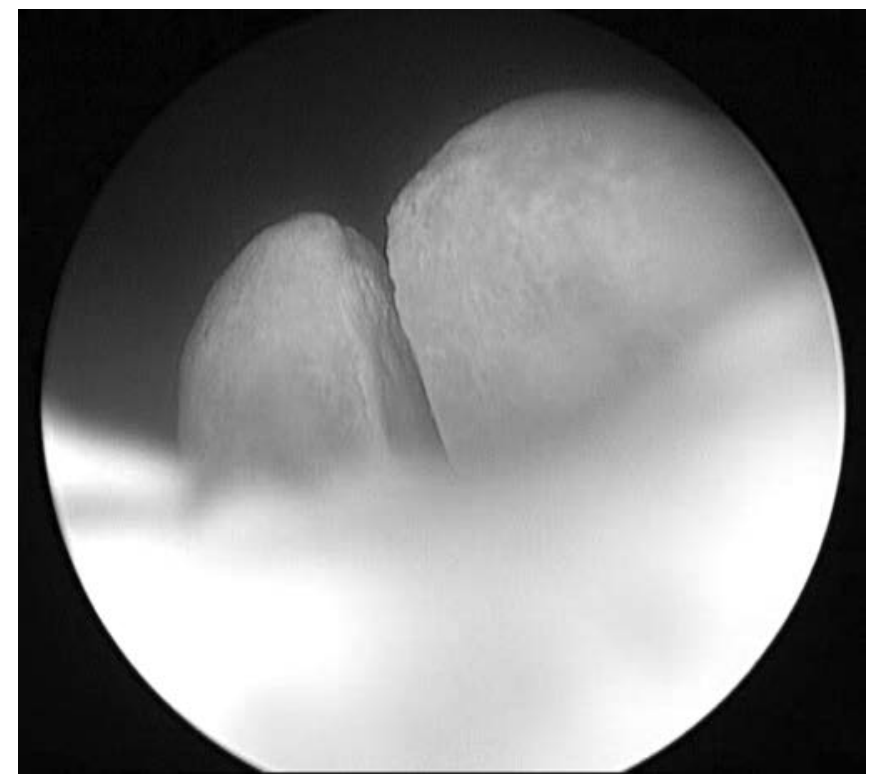

Figure 1. Cystoscopic image showing calculus within bladder.

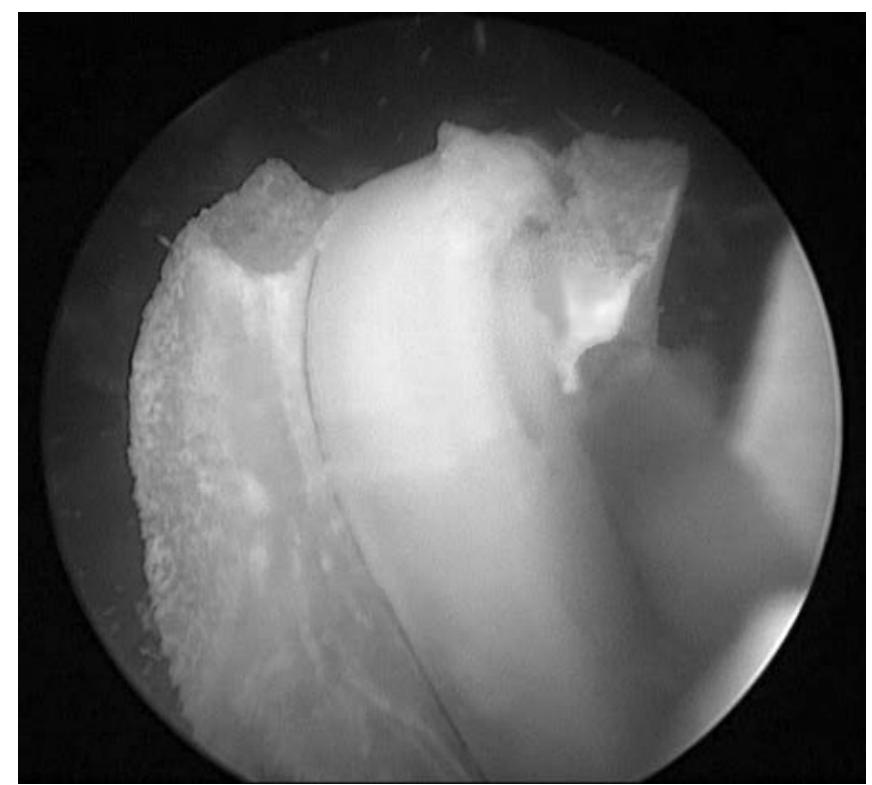

Figure 2. Hem-o-lok clip after fragmentation of calculus with laser.

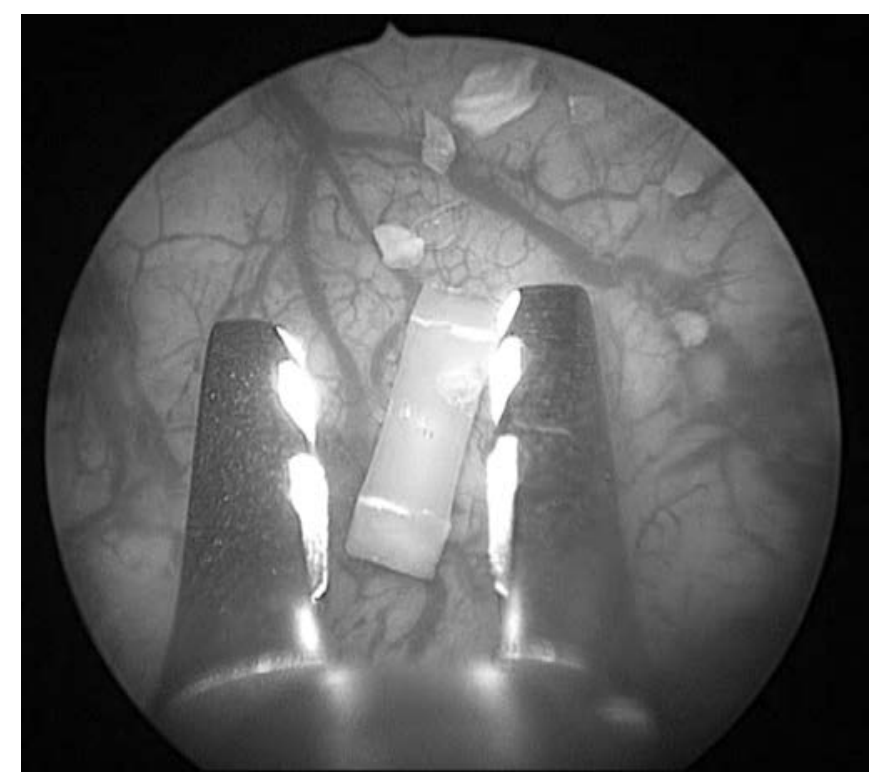

Figure 3. Incorporation of 2 Hem-o-lok clips within bladder wall.

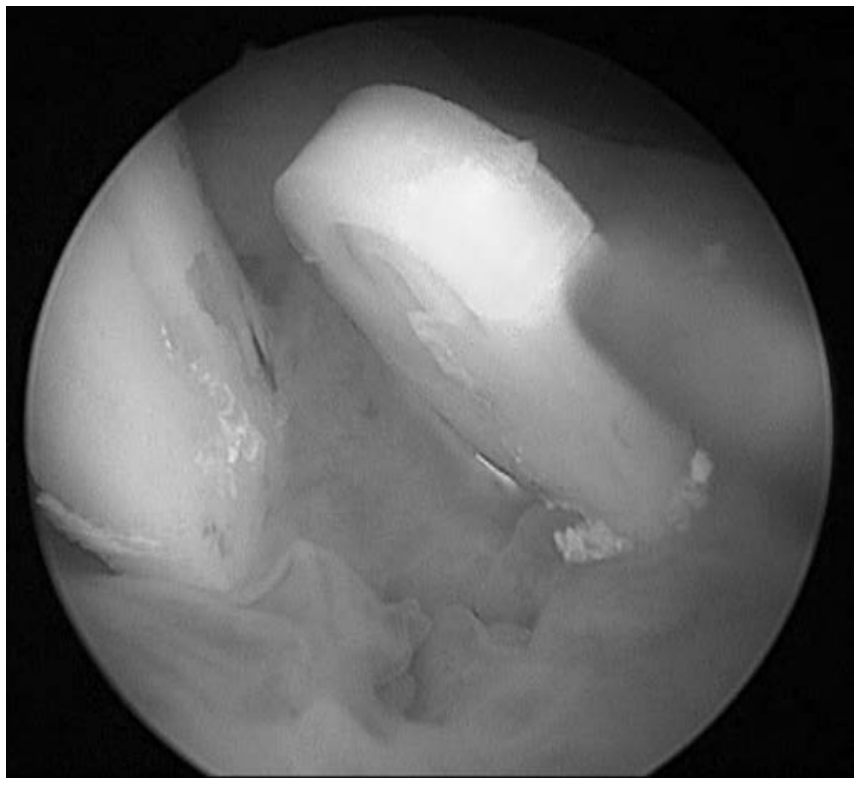

Figure 4. Removal of Hem-o-lok clips with forceps.

and transrectal ultrasonography-guided 12-core biopsy showed adenocarcinoma prostate with a Gleason score of $3+3$. The patient underwent RALRP. The bilateral vas deferens and vascular pedicles were ligated with Hem-olok clips. Urethrovesical anastomosis was performed with a single absorbable suture. The perioperative period was uneventful. The patient was discharged on the third postoperative day. The urethral catheter was removed at 2 
weeks. The patient remained asymptomatic for 3 months. After 3 months, obstructive lower urinary tract symptoms - mainly decreased flow with post-void dribblingdeveloped. The patient was evaluated and found to have vesical calculus. He then underwent urethrocystoscopy and cystolithotripsy. On cystoscopy, a free-floating intravesical Hem-o-lok clip was found to be present. The clip was removed in a transurethral manner. The urethral catheter was removed after 24 hours. The patient was asymptomatic at the most recent follow-up visit.

\section{DISCUSSION}

Minimally invasive RALRP is a widely accepted and preferred treatment option for localized prostate carcinoma with similar oncologic efficacy and low perioperative morbidity compared with open radical retropubic prostatectomy. ${ }^{1,2}$ Of the various methods used to ensure hemostasis, Hem-o-lok clip application is safe and reliable for vascular control during laparoscopic procedures. ${ }^{3}$ During RALRP, Hem-o-lok clips are used posterolaterally for control of the vascular pedicles and posteriorly for ligation of the vas deferens. The mechanisms proposed for clip migration are as follows: the clips placed over the bladder wall for hemostasis possibly became incorporated into the vesicourethral anastomosis, or perhaps the clips fell into the urinary bladder during surgery. Transvesical migration of Hem-o-lok clips can cause encrustation and further stone formation. ${ }^{4}$ Long et al ${ }^{5}$ reported vesicourethral stricture as a consequence of metal clip migration after radical prostatectomy. Blumenthal et $\mathrm{al}^{6}$ reported various complications including bladder neck contracture, erosion, and anastomotic leak due to Hem-o-lok clips that migrated into the bladder neck. The console surgeon and patientside assistant should be vigilant to avoid these complications, and any clip lying free in the pelvis or bladder should be removed before vesicourethral anastomosis. Clips, especially those used for ligation of the vas deferens, should be used minimally near the vesicourethral anastomosis. Previously, we ligated the vas deferens with clips, but we now divide the vas deferens with monopolar cautery.

\section{CONCLUSIONS}

When patients present with unexplained lower urinary tract symptoms after RALRP, one should have a high index of suspicion for possible intravesical clip migration because Hem-o-lok clips are prone to migration.

\section{References:}

1. Van Velthoven RF, Ahlering TE, Peltier A, et al. Technique for laparoscopic running urethrovesical anastomosis: the single knot method. Urology. 2003;61:699-702.

2. Schuessler WW, Schulam PG, Clayman RV, et al. Laparoscopic radical prostatectomy: initial short-term experience. Urology. 1997;50:854-857.

3. Ames CD, Perrone JM, Frisella AJ, et al. Comparison of holding strength of suture anchors for hepatic and renal parenchyma. J Endourol. 2005;19:1221-1225.

4. Banks EB, Ramani A, Monga M, et al. Intravesical Weck clip migration after laparoscopic radical prostatectomy. Urology. 2008;71:351e3-351e4

5. Long B, Bou S, Bruyere F, et al. Vesicourethral anastomotic stricture after radical prostatectomy secondary to migration of a metal clip [in French]. Prog Urol. 2006;16:384-385.

6. Blumenthal KB, Sutherland DE, Wagner KR, Frazier HA, Engel JD. Bladder neck contractures related to the use of Hemo-lok clips in robot-assisted laparoscopic radical prostatectomy. Urology. 2008;72:158-161. 